\title{
Muséologies
}

Les cahiers d'études supérieures

\section{Mirko Zardini, directeur du Centre Canadien d'architecture}

\section{Alessandra Mariani}

Volume 2, numéro 1, octobre 2007

URI : https://id.erudit.org/iderudit/1033598ar

DOI : https://doi.org/10.7202/1033598ar

Aller au sommaire du numéro

Éditeur(s)

Association Québécoise de Promotion des Recherches Étudiantes en Muséologie (AQPREM)

ISSN

1718-5181 (imprimé)

1929-7815 (numérique)

Découvrir la revue

Citer ce document

Mariani, A. (2007). Mirko Zardini, directeur du Centre Canadien d'architecture. Muséologies, 2(1), 76-83. https://doi.org/10.7202/1033598ar d'utilisation que vous pouvez consulter en ligne.

https://apropos.erudit.org/fr/usagers/politique-dutilisation/ 
Entrevue deux

Mirko Zardini,

\section{directeur du Centre Canadien d'architecture}

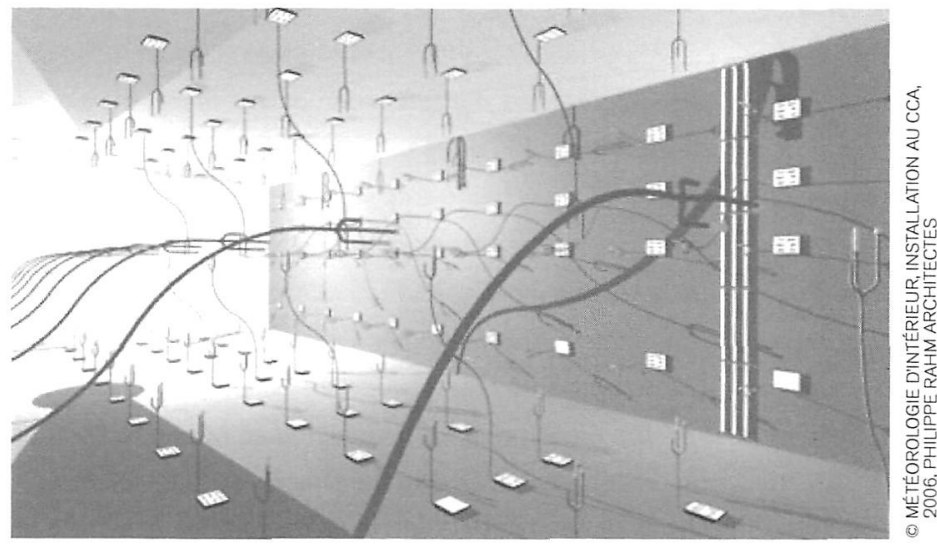

[réalisée par Alessandra Mariani le 6 septembre 2007]

Mirko Zardini est un architecte établi à Montréal et à Milan dont les recherches, les écrits et les projets de conception mettent en jeu l'architecture contemporaine, ses transformations et sa relation avec la ville et le paysage. En novembre 2005, il a été nommé directeur du Centre Canadien d'Architecture, où il a organisé des expositions telles que Sortis du cadre: Price, Rossi, Stirling + Matta-Clark, Sensations urbaines, et Désolé, plus d'essence. Ancien rédacteur en chef de Casabella (1983-1988) et de Lotus International (1988-1999), il a enseigné à l'École polytechnique fédérale suisse de Zurich (ETH) et de Lausanne (EPFL), à la GSD de Harvard, à l'Université de Venise et, à l'Université Princeton. Au nombre des ouvrages qu'il a publiés figurent notamment Paesaggi ibridi (Milan, 1996), Annähernd Perfekte Peripherie (An Almost Perfect Perception) (Bâle, Boston et Berlin, 2001) et (Milan, 2003), qui présente les résultats d'une recherche approfondie sur les caractéristiques de la ville contemporaine réalisée pour une série d'expositions inaugurée par Asphalt (Triennale de Milan, printemps 2003). 
ALESSANDRA MARIANI

Monsieur Zardini, exposez-nous votre parcours, de l'architecture à l'espace muséal.

\section{MIRKo ZARDINI}

J'ai reçu une formation traditionnelle d'architecte en Italie où la pratique est liée non seulement à l'exercice de la profession, mais aussi à l'aspect intellectuel de celle-ci. C'est pour cette raison que j'ai à la fois exercé mon métier d'architecte, poursuivi le travail de recherche et d'enseignement universitaire, collaboré à la rédaction et à l'édition de revues et d'ouvrages spécialisés en architecture et finalement agi en tant que commissaire d'expositions. Mon arrivée au Centre Canadien d'Architecture représente donc pour moi une étape importante dans ce parcours de recherche qui, aujourd'hui, est indissociable du rôle de l'architecte. Par conséquent, il ne s'agit pas, en ce qui me concerne, d'une bifurcation. Il est clair que le centre de recherche et le musée du CCA occupent tout mon temps : j'ai choisi, pour une certaine période, de mettre de côté la pratique professionnelle, mais il est évident que j'exercerai à nouveau. Pour l'instant, je participe de façon ponctuelle à des concours et à des projets de plus petite envergure.
ALESSANDRA MARIANI

Vous êtes le troisième directeur du CCA après Phyllis Lambert, mais le premier architecte pratiquant dans cette succession. Comment comptez-vous caracteriser votre direction?

\section{MIRKo ZARDINI}

$A u$ cours des vingt dernières années, Phyllis Lambert en tête, Kurt Forster et Nicholas Olsberg ont créé une situation favorable. Ils ont entre autres constitué une collection substantielle d'archives nationales et internationales, notamment les fonds qui témoignent de l'architecture des années 1960 et 1970, plus spécifiquement ceux de Cedric Price, de James Stirling, d'Aldo Rossi, de Peter Eisenman, de John Hejduk et d'Arthur Erickson. L'étendue de ces archives confirme la préoccupation du CCA de mettre à la disposition des chercheurs une importante collection d'archives d'architectes contemporains et d'ainsi enrichir le debat. Évidemment, ma formation d'architecte me permet d'énoncer des priorités différentes de celles de mes prédécesseurs. Par ma profession, je suis plus près de l'architecture contemporaine et l'orientation que je privilégie differe de celle d'autres institutions.

Nos sociétés font face à de grandes transformations et celles des derniers temps ont non seulement été intenses, mais elles se sont produites à un rythme accéléré. Elles ont engendré des réflexions marquantes sur l'organisation $d u$ territoire, sur l'environnement, sur les 
problèmes d'habitation et de la condition urbaine. La technologie et l'environnement numérique ont aussi eu un impact sur la façon d'exercer la profession d'architecte. Un sentiment d'urgence a surgi à travers des crises parfois violentes. La cité, la ville, en quête de solutions, se tourne à présent vers l'architecture, l'urbanisme, l'aménagement paysager, le design, parce que ces disciplines peuvent répondre à leurs besoins. Je crois néanmoins que, parfois, ces disciplines ne sont pas en mesure d'offrir des réponses adéquates aux demandes de la société et du monde contemporain. Mon objectif personnel est donc, ici au CCA, de favoriser le développement d'un discours, d'une réflexion, d'un projet qui puisse stimuler la recherche afin de trouver des solutions alternatives. Naturellement, cela nécessite la construction d'une nouvelle plateforme culturelle sur laquelle bâtir le discours. Cela implique aussi qu'il faut admettre qu'une certaine période de l'architecture prend fin et qu'une autre émergera inévitablement et prendra appui sur ce nouveau discours. Ce sont ces considérations qui nous ont poussés à créer l'exposition Gilles Clément / Philippe Rahm - Environnement manières d'agir pour demain ${ }^{[1]}$; qui nous ont aussi conduits à Sensations urbaines $^{[2]}$, une exposition basée sur la perception sensorielle, qui est en fait une critique de la prévalence de l'aspect purement visuel de l'architecture des vingt dernières années.
Ce sont elles, enfin, qui motivent notre prochaine exposition, 1973 : Désolé, plus d'essence ${ }^{[3]}$, qui traitera de la crise énergétique de 1973. Tous nos colloques, conférences, films, et publications servent à prolonger, à ancrer les messages véhiculés dans ces expositions. Ces réflexions, ces recherches que nous entreprenons, contribueront à construire, je l'espère, un nouveau paysage, une nouvelle scène culturelle.

[1]

Gilles Clément / Philippe Rahm -

Environnement manières d'agir pour demain.

Exposition présentée au Centre Canadien d'Architecture du 18 octobre 2006 au 10 juin 2007.

[2]

Sensations urbaines.

Exposition présentée au Centre Canadien d'Architecture du 26 octobre 2005 au 10 septembre 2006.

[3]

1973 : Désolé, plus d'essence.

L'exposition ouvrira au Centre Canadien d'Architecture le 7 novembre 2007 et sera en place jusqu'au 20 avril 2008. 
AlessandRa Mariani

Donc il s'agit ici de mener le CCA dans un rôle actif et vers un engagement social?

\section{MIRKo ZARDINI}

Oui. C'est une décision politique et c'est ce dont il s'agit. Nous croyons que chaque institution a une responsabilité intellectuelle - pour ne pas dire morale - et notre lecture de la situation contemporaine nous conduit vers cet engagement. Il y a des moments où certains problèmes doivent être abordés plutôt que d'autres. Pour nous, ce sont ceux liés aux contextes urbain et environnemental qui retiennent notre attention et nos efforts. C'est pour cette raison que nous préférons concevoir des expositions sur ces thèmes plutôt que de présenter des expositions monographiques qui ont trop souvent, dans le passé, manqué de dimension critique. Sans diminuer l'importance de telles expositions, elles semblent pour l'instant être moins en mesure de répondre à nos préoccupations.

ALESSANDRA MARIANI

La façon d'exposer l'architecture s'est considérablement transformée au cours des vingt dernières années. Comment, selon vous, cette transformation s'est-elle produite?

\section{MIRKO ZARDINI}

Les expositions d'architecture ne sont qu'une façon de communiquer les recherches qui se font dans ce domaine. Il y a entre autres les publications, les médias, le Web, qui sont des moyens de communication de plus en plus sophistiqués. Le médium exposition a beaucoup évolué si l'on se fie simplement au concept de l'architecture de l'information qu'énonce Richard Saul Wurman ${ }^{[4]}$; par exemple, on peut entrevoir queles niveaux d'accessibilitéde l'information, de sa diffusion et de sa transmission atteindront aisément des portées toujours plus grandes. Il existe maintenant des installations permanentes dans certains musées dont les composantes éducatives et informatives sont très fortes. Ces façons de faire toujours renouvelées, toujours repensées, sont liées à la crise qui sévit dans le monde muséal concernant le rôle que ce dernier a à jouer. De sa fonction de conservation et de statut de dépositaire $d u$ patrimoine universel qu'il était auparavant, il se transforme peu à peu en un lieu de transmission de l'information, d'éducation et même de récréation.

[4]

Richard Saul Wurman est un graphiste et architecte américain qui, en 1976, a élaboré la formule information architect. À l'origine des conférences TED (Technology Entertainement Design), il s'est penché sur les divers concepts de design informationnel, la puissance graphique, les technologies de l'information, les médias et les problématiques actuelles reliées à l'accès à l'information et à la surcharge cognitive. Voir : 〈http://www.wurman.com>. 
Au CCA, nous cherchons à faire de nos expositions des explorations particulières qui tentent de refléter l'état de la recherche contemporaine. Elles sont l'objet de traitements spécifiques, de stratégies de communication particulières en fonction des diverses questions et des divers contextes qui sont abordés. Ces stratégies de communication ne sont jamais les mêmes. Elles découlent des exigences issues $d u$ contenu à transmettre. C'est pour cette raison que pour l'exposition Sensations urbaines nous avons favorisé une approche complètement différente de celle utilisée pour Gilles Clément / Philippe Rahm - Environnement manières d'agir pour demain ou encore Désolé, plus d'essence, qui fut, en 1973, un message prémonitoire de ce qui semble se concrétiser aujourd'hui sur le plan énergétique. Sortis du cadre, par ailleurs, reflétait l'idée de remettre en question le caractère de permanence, de stabilité inhérente à l'exposition, fût-elle temporaire. L'idée, la stratégie adoptée a été celle de montrer un côté "évolutif", de l'apparenter à celle des archives que l'on découvre au fil du temps et, par conséquent, de remplacer les objets exposés périodiquement pendant toute la durée de l'exposition. Pour Sensations urbaines, puisque tout devait se rapporter aux perceptions sensorielles, nous devions nous abstenir d'utiliser ces dernières de façons trop mécaniques, trop systématiques, et nous devions éviter de nous engager dans une dimension sensorielle excessive. Nous avons opté pour une muséographie "classique" dans laquelle des moments de rupture, des glissements, des eléments déclencheurs venaient rompre l'aspect traditionnel de la visite de l'exposition. On pouvait toucher à du bitume, écouter des extraits sonores ou sentir des odeurs urbaines. Pour Gilles Clément / Philippe Rahm - Environnement manières d'agir pour demain, nous avons travaillé avec des installations qui se voulaient des manifestes du travail de Clément et de Rahm. Les programmes éducatifs et les visites guidées que nous offrons favorisent l'accès à ces contenus et permettent de nous rapprocher d'un public êlargi.

\section{ALESSANDRA MARIANI}

Quels moyens privilégiez-vous pour exposer l'architecture?

\section{MIRKo ZARDINI}

Qu'est-ce que l'architecture? L'architecture n'est jamais exposée. Ce sont les archives qui sont exposées; si l'on voulait exposer l'architecture, il faudrait exposer un bâtiment. Par conséquent, nous travaillons toujours avec les archives, les matériaux : comme si, pour exposer une œuvre d'art, nous présentions les eléments qui ont servi à la créer ou les outils qui ont servi à la communiquer. Une exposition d'architecture differe, dans les manières de la présenter, d'une exposition d'œuvres d'art dont les œuvres se suffisent à elles-mêmes. 
L'original - le bâtiment - n'est jamais exposé : ce sont des dessins, des coupes, des elévations, puis son rendu par des photographies, quel'on présente. Ce sont aussi des films ou des vidéos sur les usages que l'on fait de ce bâtiment qui sont projetés. Il s'agit donc d'une représentation de quelque chose qui est toujours manquant. La stratégie quej'aime adopter est celle d'être plus ouvert à la diversité des matériaux. C'est pourquoi la collection du CCA, plutôt que de ne conserver qu'un ou deux dessins d'un projet, privilégie l'acquisition des archives entières parce qu'elles renferment le processus complet de la productiond'un bâtiment ou d'un projet de réaménagement urbain. Ces objets sont les témoins de tous ceux qui ont collaboré à un projet donné. Dès lors, les archives deviennent à leur tour un échantillon de la société qui produit une œuvre d'architecture. Puisque l'acquisition de nos archives est basée sur cette idée, nos expositions le sont également.

De plus, bien que temporaire, l'aménagement de chacune des expositions constitue un acte architectural en soi.C'est la raison pour laquelle nous portons une si grande attention à cette facette de chacun des projets que nous réalisons, nous assurant de faire le lien avec le thème et le contenu de l'exposition.
ALESSANDRA MARIANI

Nous assistons présentement à un engouement pour l'interdisciplinarité. Est-ce selon vous productif : voyonsnous vraiment un élargissement de la médiation des messages, des propos, des discours?

MIRKo ZARDINI

Pour répondre à cette question, permettez-moi de m'exprimer en termes d'architecture. Il y a eu des moments où l'architecture était tournée sur ellemême, comme discipline autonome, et il y a des moments où l'architecture sintéresse aux autres disciplines, comme c'est le cas présentement. je crois que la première chose que tout architecte se doit de faire quotidiennement est de lire les journaux, de naviguer sur le Net pour comprendre l'univers dans lequel il vit. Aussi, lorsqu'il s'agit d'entreprendre une recherche ou d'exposer l'architecture, d'autres disciplines peuvent apporter une contribution fort importante qui enrichit et crée de nouvelles clés d'interprétation et de communication.

\section{ALESSANDRA MARIANI}

S'il y a lieu, ces propos sont-ils adoptés par le CCA?

MIRKO ZARDINI

Le CCA favorise la fréquentation d'autres disciplines parce que ces interrelations s'avèrent toujours très fructueuses. Elles offrent la capacité d'aller plus loin dans nos efforts de recherche, de communication et de médiation muséale. Si nous présentons une série de films sur la crise énergétique, par exemple, ceux-ci peuvent intéresser un autre public et lui présenter d'autres angles pour aborder la problématique que nous traitons. 


\section{ALESSANDRA MARIANI}

Sensations urbaines : l'exposition et son catalogue ont bénéficié d'une certaine fortune critique. Croyez-vous que la muséologie d'immersion puisse concrètement transmettre un savoir?

MIRKO ZARDINI

Il faut avouer que cette exposition n'a pas eu très bonne presse dans le milieu architectural local. Dans l'ensemble, toutefois, la critique a été favorable. Parce que l'exposition ne comportait pas vraiment de représentations architecturales, ceux qui ont émis des réserves ont invoqué le fait qu'il ne s'agissait pas d'une exposition d'architecture. À un certain degré, ils avaient raison, parce que l'intention initiale était d'exposer une critique de la pensée architecturale contemporaine et de ce courant actuel qui surenchérit l'aspect visuel. Nous voulions introduire le concept qu'il existe une qualité sensorielle inhérente aux espaces que nous produisons et que nous devons entretenir. Ces réflexions étaient déjà amorcées dans des études, notamment en anthropologie, en histoire sociale et en géographie. Je trouvais personnellement curieux que ces réflexions qui avaient cours depuis un certain temps dans ces disciplines n'avaient pas encore trouvé écho en architecture et en urbanisme, où ces composantes sensorielles sont pourtant essentielles à l'expérience que nous faisons de l'environnement bâti et urbain. Nous voulions proposer aux visiteurs d'aborder avec un regard différent la qualité de l'environnement dans lequel ils vivent. Nous souhaitions leur offrir la possibilité de se forger une opinion sur leur environnement d'une façon un peu plus sophistiquée et de formuler une critique autre que celle qui est communément diffusée. Encesens, l'exposition aétéun succès. Les expositions à venir font partie d'une suite logique d'idées, mais nous allons sans aucun doute profiter de l'expérience acquise à l'occasion de l'exposition Sensations urbaines.

\section{ALESSANDRA MARIANI}

Le CCA compte un des centres de recherche les plus en vue du monde muséal. Au moment où la plupart des institutions désistent la recherche au profit des stratégies de communication et de mise en marché, expliquez-nous comment vous bénéficiez de ce centre de recherche.

MIRKO ZARDINI

Afin de s'assurer que les objectifs seront atteints, il faut les mesurer au regard de la mission d'une institution. Notre mission nous définit principalement comme un centre de recherche et, ensuite, comme un musée. La recherche est donc la composante première de notre institution. Elle connait parfois du succès, parfois moins : c'est inévitable. Nous avons le sentiment que notre mission consiste en la poursuite d'investigations dans le but de contribuer au développement des connaissances en architecture et en environnement social (qui comprend l'architecture, l'urbanisme, l'aménagement paysager, etc.). Le résultat de ces recherches alimente nos discours et, conséquemment, 


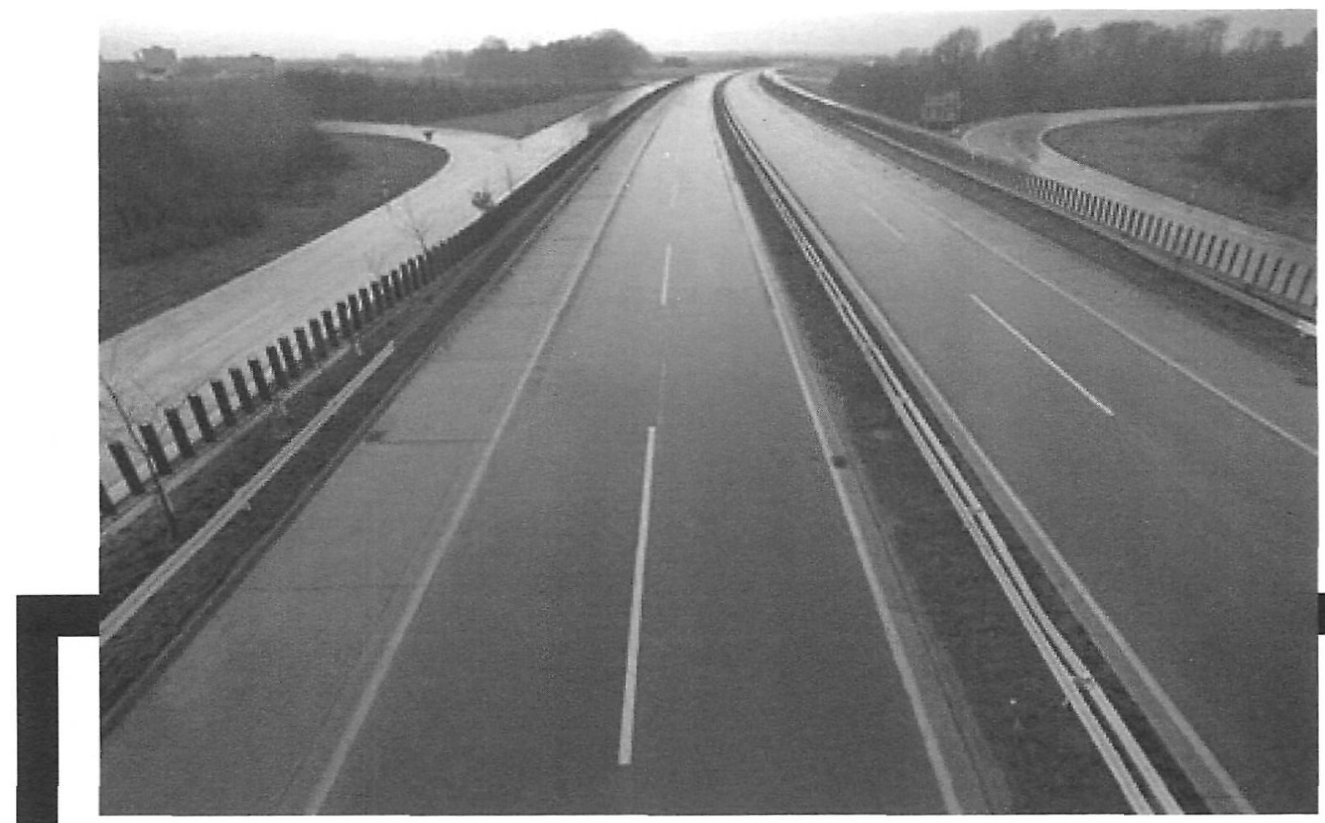

Düsseldorf-Wuppertal échangeur lors d'interdiction de conduite, Allemagne, 15 novembre 1973. 1973 : désolé, plus d'essence, une exposition présentée au Centre Canadien d'Architecture du 7 novembre 2007 au 20 avril 2008.

transforme notre façon d'aborder les expositions. Le centre de recherche est pour nous une fenêtre sur le monde. Nous y accueillons des chercheurs de divers pays qui partagent avec nous leurs idées. Le dialogue que nous entretenons avec eux contribue évidemment à l'élaboration de nouveaux discours et nous souhaitons que ce dialogue puisse s'accrôtre àl'avenir.

\section{ALESSANDRA MARIANI}

Selon vous, quels changements, quelles transformations sont présentement nécessaires au paysage muséal québécois?

\section{MIRKO ZARDINI}

Les discussions que j'ai eues à ce jour avec mes collègues du milieu me confirment que d'importants efforts sont déployés dans le but d'assurer un solide réseau des musées. Toutefois, il serait avantageux de canaliser ces énergies pour élaborer une stratégie commune visant à favoriser des échanges avec d'autres institutions dans le monde. Que les chercheurs soient à Montréal, à New York ou ailleurs, ils doivent comprendre que ce genre d'échanges peut créer des occasions uniques. Aujourd'hui les possibilités sont multiples et les échanges culturels enrichissent notre communauté. Mon message est simple : regardons plus loin, ailleurs, ouvrons-nous surle monde. 